Fl uct uat $i$ on of adenosi ne concent $r$ at $i$ on by modes of intravenous inf usi on based on mat hemat i cal si mul at $i$ on and exper i ments

\begin{tabular}{|l|l|}
\hline 著者 & $\begin{array}{l}\text { Nakaj i ma Keni chi, Taki Juni chi, Yoneyanæ } \\
\text { Tat suya, Fukuoka Nakot o, Kayano Dai ki , Tonami } \\
\text { Nori hi sa }\end{array}$ \\
\hline $\begin{array}{l}\text { j our nal or } \\
\text { publ i cat i on t i t l e }\end{array}$ & Annal s of Nucl ear Nedi ci ne \\
\hline vol une & 20 \\
\hline nunber & 7 \\
\hline page range & $485-491$ \\
\hline year & $2006-01-01$ \\
\hline URL & ht t p: //hdl . handl e. net /2297/2458 \\
\hline
\end{tabular}




\title{
Fluctuation of adenosine concentration by modes of intravenous infusion based on mathematical simulation and experiments
}

\author{
Kenichi NaKajima, Junichi Taki, Tatsuya Yoneyama, Makoto Fukuoka, \\ Daiki KaYAno and Norihisa TONAMI
}

Department of Nuclear Medicine, Kanazawa University Hospital

\begin{abstract}
Objective: Adenosine, which has been used for a myocardial perfusion scan, shows rapid clearance from blood because of its short half-life of $<10$ seconds. This simulation study evaluates influences of modes of radionuclide injection on ventricular adenosine concentration when one intravenous injection line is used. Methods: Assuming that radionuclide injection is a unit impulse, time-activity curves were measured in the left ventricle (LV) and fitted by a gamma function. Typical patterns of concentration fluctuation when adenosine infusion was temporarily modified were calculated by the convolution integral of input function and unit impulse response. Variation of concentration was measured by experiments using continuous ${ }^{99 \mathrm{~m}} \mathrm{Tc}$ injection and co-infusion of water via a three-way stopcock. Modes of co-infusion with various infusion speeds and volumes were examined. Results: Intermission of adenosine infusion and rapid displacement by radionuclide co-injection significantly influenced the adenosine concentration in LV. Intermission of adenosine infusion for 2 seconds caused a 15\% decrease in the adenosine concentration in the left ventricle. When a square-shaped input was assumed, a three-fold higher concentration of adenosine for 3 seconds created by radionuclide injection resulted in a $+42 \%$ increase in the $\mathrm{LV}$ concentration. Based on a measured input function, radionuclide injection using three-way stopcock through one route caused a two- to three-fold increase in the steady concentration in the vein just after injection. When $0.5 \mathrm{~m} l$ of radionuclide was slowly co-injected, with three ways opened, it caused a relatively low fluctuation, creating a $+34 \%$ to $-47 \%$ change in concentration of $\mathrm{LV}$. A flush of radionuclide with physiological saline significantly increased the adenosine concentration in LV, when short half-lives were assumed. Conclusion: An intravenous adenosine and radiopharmaceutical injection in the same line is feasible. However, the fluctuation of concentration depends significantly on the mode of injection. To minimize the fluctuation, a slow injection of a small volume of a myocardial imaging agent via a co-injection route, with three ways opened, is recommended.
\end{abstract}

Key words: adenosine, myocardial perfusion imaging, adverse effect, simulation

\section{INTRODUCTION}

PharmaCological STRESS TESTING in a myocardial scan is an important option for patients unable to exercise, and dipyridamole and adenosine triphosphate (ATP) have been used in Japan. While adenosine has been widely used in the US and Europe, ${ }^{1}$ it has become available in Japan

Received March 6, 2006, revision accepted June 18, 2006.

For reprint contact: Kenichi Nakajima, M.D., Department of Nuclear Medicine, Kanazawa University Hospital, Kanazawa 920-9641, JAPAN.

E-mail:nakajima@med.kanazawa-u.ac.jp only recently in $2005,{ }^{2-4}$ and the use of this vasodilator drug will increase. The use of two intravenous lines for adenosine and radiopharmaceuticals is generally recommended, but injection through a single line, namely continuous adenosine injection with co-infusion of ${ }^{201} \mathrm{Tl}$ or ${ }^{99 \mathrm{~m}} \mathrm{Tc}$ perfusion imaging agents at 3 minutes, has also been used for the sake of convenience. In the setting of the single-route injection, intermission and displacement of adenosine in the infusion line may cause significant fluctuation in the concentration in the left ventricle (LV) or coronary arteries. However, actual serial variation in adenosine concentration in LV cannot be easily measured in humans. Thus, the purpose of this study is to estimate 


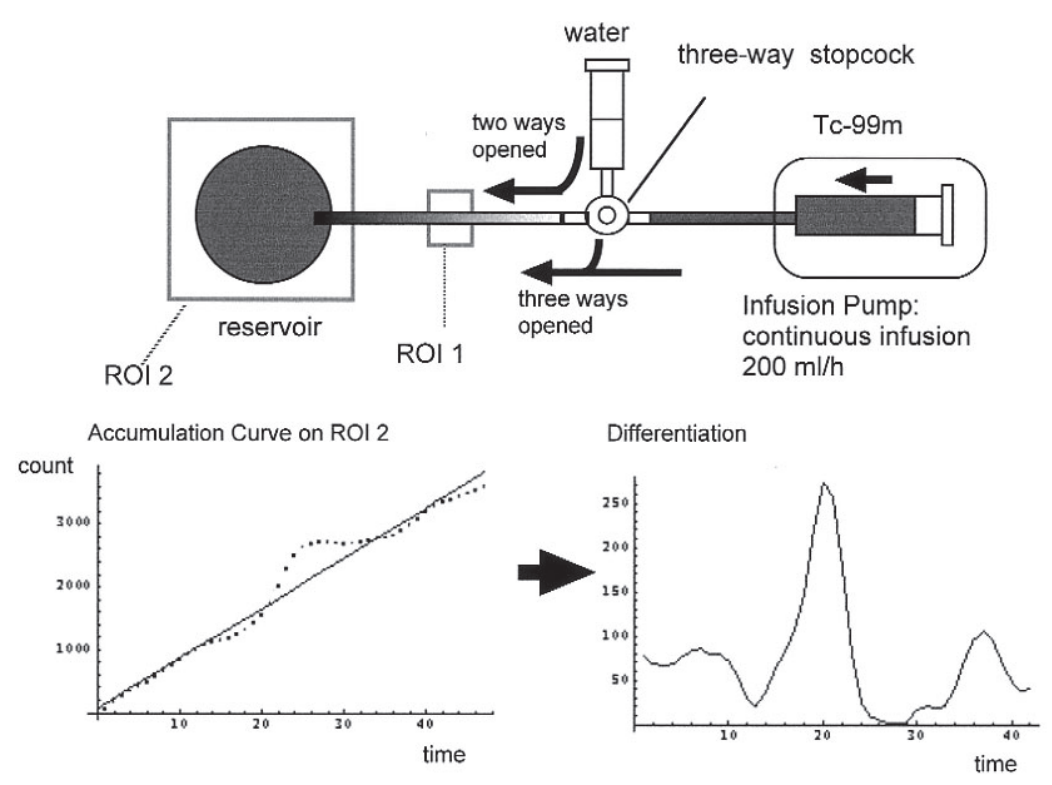

Fig. 1 Infusion of ${ }^{99 \mathrm{~m}} \mathrm{Tc}$ and co-injection of water. The accumulation curve was measured on the reservoir and the differentiation can generate the input function. The three-way stopcock was used as two ways or three ways opened as indicated by the arrows.

the fluctuation of concentration depending on the modes of adenosine and radiopharmaceutical injection. Moreover, fundamental knowledge regarding the effect of input and transfer function on the concentration in LV helps us understand the adequacy of co-injection through a single route.

\section{MATERIALS AND METHODS}

\section{Principles}

When a radionuclide and drugs are injected into the antecubital vein, they pass through the arm vein, right ventricle, lungs, LV and coronary arteries to reach the myocardium. The time-activity curves on a specific region show gradual widening and lower peaks during transit. If temporal changes in concentration in the injection site are given, and transfer function from the arm to LV is measured, the concentration in the LV can be estimated mathematically. Namely, if input is defined as a delta function, and unit impulse response $h(t)$ is known as a function of transfer, then the output will be calculated by the convolution integral of input $f(t)$ and $h(t): f(t)\left(^{*}\right)$ $h(t)[(*)$ indicates convolution integral].

In this experimental study, since we could not directly measure the serial adenosine concentration, adenosine concentration was substituted by radionuclide activity, and injected radiopharmaceutical was substituted by non-labeled water. Therefore, the condition in which continuous adenosine infusion was modified by the coinjection of radiotracer was simulated by that in which continuous ${ }^{99 \mathrm{~m}} \mathrm{Tc}$ pertechnetate infusion was modified by the co-injection of water. In this manuscript, experimental ${ }^{99 \mathrm{~m}} \mathrm{Tc}$ and water correspond to adenosine and radio- pharmaceuticals $\left({ }^{99 \mathrm{~m}} \mathrm{Tc} \mathrm{MIBI}\right.$, tetrofosmin and $\left.{ }^{201} \mathrm{Tl}\right)$, respectively. The connection of syringe, infusion lines, three-way stopcocks and reservoir is shown in Figure 1. To clearly present the model, the term "infusion" is used for continuous injection of ${ }^{99 \mathrm{~m}} \mathrm{Tc}$ pertechnetate, and the term "co-injection" for water via a three-way stopcock.

\section{Estimation of input function}

To measure ${ }^{99 \mathrm{~m}} \mathrm{Tc}$ input function from the infusion pump, the changes in count $a(t)$ (count/sec) were determined by the product of concentration at time $t$ in the infusion line $(B(t))($ count $/ \mathrm{m} l)$ and injection flow $F(\mathrm{~m} l / \mathrm{sec}): a(t)=F^{*}$ $B(t)$ [* indicates multiplication]. Although the concentration of ${ }^{99 \mathrm{~m}} \mathrm{Tc}$ at time $t$ can be measured by the region of interest (ROI) 1 in Figure 1, the flow during manual injection cannot be accurately determined. However, since the accumulated reservoir count $q$ can be measured, the rate of change (count/sec) can be calculated by the first derivative $d q / d t$. Therefore, we decided to measure the count in ROI 2. When we co-injected water through a three-way stopcock, both temporary intermission or reduction in ${ }^{99 \mathrm{~m}} \mathrm{Tc}$ count and rapid displacement took place, followed by return to a steady state.

\section{Unit impulse response}

A time-activity curve in the LV was measured using radionuclide angiography performed for a cardiac study in normal LV function. Although it was impossible to achieve complete bolus injection as a delta function, a small amount of radionuclide $<0.5 \mathrm{~m} l$ was injected as a good bolus, and the time-activity curve in the outflow of LV was recorded. This curve was fitted by the gamma function, 


$$
y=\left(t^{\wedge} a\right) * c^{*} \exp (-t / b)
$$

where $a, b$ and $c$ are coefficients of the gamma function, and recirculation was excluded. Similarly, in a patient with heart failure (output $1555 \mathrm{ml} / \mathrm{min} / \mathrm{m}^{2}$, ejection fraction $20 \%$ ), the time-activity curve was obtained. These two gamma function curves were used as typical unit impulse response functions.

\section{Definition of time-activity curves in $L V$}

Although the time-activity curve of the LV was defined by equation 1 , adenosine was supposed to show decay during transit. The right-hand side of equation 1 was then multiplied by $0.5^{\wedge}\left(T_{\mathrm{LV}} / T_{1 / 2}\right)$ as a decay correction factor (DCF):

$$
y=\left(t^{\wedge} a\right) * c^{*} \exp (-t / b) / A * \mathrm{DCF},
$$

where $T_{\mathrm{LV}}$ is an average arrival time to $\mathrm{LV}$ and assumed to be 10 seconds for normal output and 15 seconds for heart failure. $T_{1 / 2}$ is an effective half-life of adenosine, which is assumed to be 10 seconds. The term $A$ is a correction factor to make an integral of gamma function from $t=0$ to infinite 1 .

\section{Flushing by physiological saline}

The effect of flushing radionuclide using physiological saline $(10 \mathrm{~m} l)$ displaced adenosine in the tube and veins, and the arrival time to LV may have been shortened. In this flushing model, although changes in concentration in ROIs 1 and 2 flushed with saline could be measured, the shortening of the arrival time to LV could not be measured. We thus assumed that flushing by physiological saline shortened the arrival time by 5 seconds at maximum, and the degree of shortening was smaller as it approached LV. Based on the small volume of ordinarily injected myocardial imaging agent, we assumed that the arrival time to LV was not altered without flushing.

\section{Data analysis}

All the data analysis mentioned above was obtained by Mathematica Version 5.2 (Wolfram Research, Inc.). For fitting the time activity curves, a gamma function was used. For noise reduction in the time-activity curve, weighted temporal smoothing was used. Differentiation of the curve was performed by a 5-point formula of the numerical differentiation method. The convolution was performed by convolution of a kernel and list using $\Sigma_{r} K_{r} a_{s-r}$. Since fluctuation of the activity shows one or more negative or positive peaks, changes of fluctuation were expressed from the steady condition. The duration of significant fluctuation was defined as $>+20 \%$ or $-20 \%$ from the steady condition. When multiple peaks were observed, the time from the initial to the last fluctuation was calculated.
Fundamental patterns of input function and time-activity curve in $L V$

The effect of the input function on the LV curve was calculated using a basic square-shaped input function. Effects of intermission of continuous injection and duration of increase or decrease in concentration were analyzed.

Experiment 1: Rate of injection of myocardial imaging tracers

To examine the rate of injection, the following four modes were used. A syringe containing $300 \mathrm{MBq}$ of ${ }^{99 \mathrm{~m}} \mathrm{Tc}$ in $25 \mathrm{ml}$ was prepared and injected at the constant infusion rate of $200 \mathrm{~m} l / \mathrm{h}(0.055 \mathrm{~m} / / \mathrm{sec})$. (1) Two $\mathrm{m} l$ of water was co-injected for 1 second from the three-way stopcock with two ways opened. Namely, ${ }^{99 \mathrm{~m}} \mathrm{Tc}$ infusion was temporarily stopped by the stopcock, when the water was coinjected. Thereafter, the stopcock was returned to the original position, and ${ }^{99 \mathrm{~m}} \mathrm{Tc}$ flow returned to the constant flow rate again. (2) Two $\mathrm{ml}$ of water was co-injected for 1 second from the three-way stopcock with three ways opened. ${ }^{99 \mathrm{~m}} \mathrm{Tc}$ infusion was continued during co-injection. (3) Two $\mathrm{m} l$ of water was co-injected slowly for 5 seconds, which was otherwise similar to the situations in 1 and 2. (4) Two $\mathrm{m} l$ of water was co-injected very slowly for 10 seconds, which was otherwise similar to the situations in 1 and 2.

Experiment 2: Volume and mode of injection with three ways opened

In the same setting as that of experiment 1 , the volume of water co-injection was changed to $0.5,1,2$ and $3 \mathrm{~m} l$ with three ways opened, and it was not flushed with saline. In addition, $1 \mathrm{~m} l$ of a water was co-injected slowly for 5 seconds. No intermission of ${ }^{99 \mathrm{~m}} \mathrm{Tc}$ infusion occurred during water co-injection.

Experiment 3: Volume and mode of injection with two ways opened

This experiment was performed to evaluate the change in concentration when two ways of the three-way stopcock was opened. In ordinary injection through the threeway stopcock, the continuous flow is transiently stopped during co-injection of radiopharmaceuticals. After the injection is finished, continuous injection is restarted. To simulate this condition, we used a water volume of $0.5,1$, 2 and $3 \mathrm{~m} l$ with bolus injection, which was not flushed with saline.

Experiment 4: Flushing with physiological saline

When adenosine injection was followed by flushing with a large amount of physiological saline, it may have reached the coronary artery with the higher concentration. The change in concentration was measured after flushing of $10 \mathrm{~m} l$ of water. Although the flushing would shorten the arrival time to the $\mathrm{LV}$, the effect of flushing could not be 
Table 1 Fundamental characteristics of simulated adenosine injection and concentration in the left ventricle

\begin{tabular}{|c|c|c|c|c|}
\hline \multirow[b]{2}{*}{ Simulated situation } & \multicolumn{2}{|c|}{ Normal output } & \multicolumn{2}{|c|}{ Heart failure } \\
\hline & Peak & $\begin{array}{l}\text { Duration of } \\
\text { fluctuation* }\end{array}$ & Peak & $\begin{array}{l}\text { Duration of } \\
\text { fluctuation* }\end{array}$ \\
\hline \multicolumn{5}{|l|}{ Intermission of infusion } \\
\hline intermission $1 \mathrm{sec}$ & $93 \%$ & 0 & $95 \%$ & 0 \\
\hline intermission $2 \mathrm{sec}$ & $86 \%$ & 0 & $90 \%$ & 0 \\
\hline intermission $5 \mathrm{sec}$ & $66 \%$ & 13 & $76 \%$ & 10 \\
\hline intermission $10 \mathrm{sec}$ & $46 \%$ & 19 & $54 \%$ & 23 \\
\hline \multicolumn{5}{|c|}{ Concentration and duration of changes } \\
\hline$\times 0.2,3 \mathrm{sec}$ & $83 \%$ & 0 & $88 \%$ & 0 \\
\hline$\times 0.5,3 \mathrm{sec}$ & $90 \%$ & 0 & $93 \%$ & 0 \\
\hline$\times 2.0,3 \mathrm{sec}$ & $121 \%$ & 4 & $115 \%$ & 0 \\
\hline$\times 3.0,3 \mathrm{sec}$ & $142 \%$ & 14 & $129 \%$ & 15 \\
\hline$\times 3.0,1 \mathrm{sec}$ & $114 \%$ & 0 & $110 \%$ & 0 \\
\hline$\times 3.0,3 \mathrm{sec}$ & $142 \%$ & 14 & $129 \%$ & 15 \\
\hline$\times 3.0,5 \mathrm{sec}$ & $168 \%$ & 19 & $148 \%$ & 23 \\
\hline$\times 3.0,7 \mathrm{sec}$ & $193 \%$ & 21 & $166 \%$ & 25 \\
\hline$\times 3.0,9 \mathrm{sec}$ & $215 \%$ & 24 & $184 \%$ & 30 \\
\hline
\end{tabular}

The steady concentration is defined as $100 \%$. defined as out of $\pm 20 \%$ from the first to the last peak

accurately estimated. Then we assumed that the arrival time to LV was shortened by 5 seconds, and the effect of shortening was linearly decreased within 10 seconds after injection. The effective half-life of adenosine was assumed to be 1, 2, 5 and 10 seconds. ${ }^{5,6}$

\section{RESULTS}

\section{Fundamental patterns after injection}

When a square-shaped input function was used, the time activity curve reached a plateau in 30 seconds in the normal patient and in 35 seconds in the heart-failure patient after the time point of $1 \%$ of the injected activity appeared in the LV (Fig. 2). When the arrival time from the arm to the $\mathrm{LV}$ was assumed to be 10 to 20 seconds, the activity became a constant 40 to 60 seconds after intravenous injection. After the cessation of the intravenous injection, the activity was zero 40 to 60 seconds later, depending on the cardiac function.

\section{Intermission of adenosine injection}

Switching the passage of injection routes in the three-way stopcock temporarily interrupted input of adenosine. As shown in Table 1, intermission of 1, 2, 5 and 10 seconds resulted in a decrease in concentration to $93 \%, 86 \%, 66 \%$ and $46 \%$, respectively. Duration of an intermission in 2 seconds did not generate a significant drop $(<80 \%)$ in concentration, but 5 and 10 second durations generated a decreased concentration for 13 and 19 seconds in LV. In the heart-failure condition, the degree of decrease was less significant compared with that of the normal patient.

\section{Fluctuation of adenosine concentration}

Changes in concentration for 3 seconds from 0.2 -fold to


Fig. 2 A square-shaped input function and the simulated concentration curve in the LV by the convolution integral processing. The lower panel indicates the gamma-functions showing normal and heart failure conditions.

3 -fold resulted in changes of $83 \%$ to $142 \%$ in LV. A threefold concentration, as seen in rapid adenosine infusion, for 1 to 9 seconds resulted in a $114 \%$ to $215 \%$ increase in concentration in LV. The duration of fluctuation was 14 to 24 seconds. The degree of fluctuation was less in the heartfailure patient.

\section{Experiment 1: Injection speed}

An injection of two $\mathrm{m} l$ of water was used in this experiment. When the concentration curve was observed, the concentration just after injection showed a 2 to 3 -fold 
Table 2 Simulated adenosine injection and concentration in the left ventricle in 4 experiments

\begin{tabular}{|c|c|c|c|c|c|c|c|c|}
\hline \multirow[b]{2}{*}{ Simulated situation } & \multicolumn{4}{|c|}{ Normal output } & \multicolumn{4}{|c|}{ Heart failure } \\
\hline & $\begin{array}{l}\text { First } \\
\text { peak }\end{array}$ & $\begin{array}{c}\text { Second } \\
\text { peak }\end{array}$ & $\begin{array}{l}\text { Third } \\
\text { peak }\end{array}$ & $\begin{array}{l}\text { Duration of } \\
\text { fluctuation* }\end{array}$ & $\begin{array}{l}\text { First } \\
\text { peak }\end{array}$ & $\begin{array}{l}\text { Second } \\
\text { peak }\end{array}$ & $\begin{array}{l}\text { Third } \\
\text { peak }\end{array}$ & $\begin{array}{l}\text { Duration of } \\
\text { fluctuation* }\end{array}$ \\
\hline \multicolumn{9}{|l|}{ Experiment 1} \\
\hline 2-way, Intermission $1 \mathrm{sec}$, adenosine $2 \mathrm{~m} / / 1 \mathrm{sec}$ & $89 \%$ & $149 \%$ & $86 \%$ & $11 \mathrm{sec}$ & $95 \%$ & $127 \%$ & $94 \%$ & $9 \mathrm{sec}$ \\
\hline 3 -way, No intermission, adenosine $2 \mathrm{~m} / / 1 \mathrm{sec}$ & $115 \%$ & $69 \%$ & & $11 \mathrm{sec}$ & $107 \%$ & $79 \%$ & & $6 \mathrm{sec}$ \\
\hline 3-way, No intermission, adenosine $2 \mathrm{ml} / 5 \mathrm{sec}$ & $145 \%$ & $64 \%$ & & $24 \mathrm{sec}$ & $128 \%$ & $79 \%$ & & $27 \mathrm{sec}$ \\
\hline 3-way, No intermission, adenosine $2 \mathrm{~m} / / 10 \mathrm{sec}$ & $134 \%$ & $49 \%$ & & $32 \mathrm{sec}$ & $121 \%$ & $65 \%$ & & $32 \mathrm{sec}$ \\
\hline \multicolumn{9}{|l|}{ Experiment 2: 3 ways opened } \\
\hline adenosine $0.5 \mathrm{ml}$, bolus & $163 \%$ & $91 \%$ & & $16 \mathrm{sec}$ & $143 \%$ & $97 \%$ & & $18 \mathrm{sec}$ \\
\hline adenosine $1 \mathrm{ml}$, bolus & $173 \%$ & $76 \%$ & & $28 \mathrm{sec}$ & $148 \%$ & $89 \%$ & & $17 \mathrm{sec}$ \\
\hline adenosine $2 \mathrm{~m} l$, bolus & $177 \%$ & $67 \%$ & & $31 \mathrm{sec}$ & $150 \%$ & $82 \%$ & & $17 \mathrm{sec}$ \\
\hline adenosine $3 \mathrm{~m} l$, bolus & $168 \%$ & $63 \%$ & & $32 \mathrm{sec}$ & $143 \%$ & $77 \%$ & & $33 \mathrm{sec}$ \\
\hline adenosine $1 \mathrm{~m} l$, slow infusion & $134 \%$ & $53 \%$ & & $31 \mathrm{sec}$ & $123 \%$ & $69 \%$ & & $29 \mathrm{sec}$ \\
\hline \multicolumn{9}{|l|}{ Experiment $3: 2$ ways opened } \\
\hline adenosine $0.5 \mathrm{ml}$, bolus & $97 \%$ & $135 \%$ & $80 \%$ & $9 \mathrm{sec}$ & - & $121 \%$ & $91 \%$ & $5 \mathrm{sec}$ \\
\hline adenosine $1 \mathrm{~m} l$, bolus & $99 \%$ & $151 \%$ & $82 \%$ & $12 \mathrm{sec}$ & - & $132 \%$ & $92 \%$ & $12 \mathrm{sec}$ \\
\hline adenosine $2 \mathrm{~m} l$, bolus & $97 \%$ & $160 \%$ & $92 \%$ & $13 \mathrm{sec}$ & - & $138 \%$ & $98 \%$ & $15 \mathrm{sec}$ \\
\hline adenosine $3 \mathrm{ml}$, bolus & $70 \%$ & $153 \%$ & $91 \%$ & $25 \mathrm{sec}$ & - & $129 \%$ & $98 \%$ & $11 \mathrm{sec}$ \\
\hline \multicolumn{9}{|c|}{ Experiment 4 : flushed with $10 \mathrm{ml}$ of water, 3 ways opened } \\
\hline half life of adenosine $10 \mathrm{sec}$ & $127 \%$ & $78 \%$ & & $24 \mathrm{sec}$ & $117 \%$ & $84 \%$ & & $0 \mathrm{sec}$ \\
\hline half life of adenosine $5 \mathrm{sec}$ & $157 \%$ & $82 \%$ & & $14 \mathrm{sec}$ & $138 \%$ & $88 \%$ & & $15 \mathrm{sec}$ \\
\hline half life of adenosine $2 \mathrm{sec}$ & $327 \%$ & $88 \%$ & & $25 \mathrm{sec}$ & $262 \%$ & $90 \%$ & & $32 \mathrm{sec}$ \\
\hline half life of adenosine $1 \mathrm{sec}$ & $1354 \%$ & $93 \%$ & & $34 \mathrm{sec}$ & $1001 \%$ & $90 \%$ & & $46 \mathrm{sec}$ \\
\hline
\end{tabular}

* defined as out of $\pm 20 \%$ from the first to the last peak

increase in the vein (Fig. 3). When two ways of three-way stopcock were opened with 1 second of intermission and $2 \mathrm{~m} l$ of water co-injection, ${ }^{99 \mathrm{~m}} \mathrm{Tc}$ activity decreased to $89 \%$, then increased to $149 \%$, and decreased again to $86 \%$. In contrast, when water was co-injected with three ways opened, ${ }^{99 \mathrm{~m}} \mathrm{Tc}$ was flushed by the water and the concentration was temporarily increased and then it decreased. In this condition, when water was injected rapidly (1 second), slowly (5 seconds) and very slowly (10 seconds), the first peak of increase was 115 to $145 \%$, and the second decreased peak was 49 to $69 \%$. A very slow injection prolonged the duration of fluctuation.

Experiment 2: Volume and modes of injection with three ways opened

In this experiment, the water was co-injected as a bolus, and the volume was changed (Table 2). This injection temporarily increased the concentration in LV, caused by the displacement of water, and then the concentration was decreased (Fig. 3A). When the volume of 0.5 to $3 \mathrm{~m} l$ was co-injected, the initial peak was 163 to $177 \%$ and the second decrease was 63 to $91 \%$. The duration of fluctuation was shortened by $0.5 \mathrm{~m} l$ of water injection, but was 30 seconds for 1 to $3 \mathrm{~m} l$ of injection. In the heart-failure condition, the peak of fluctuation was less but the duration was 17 to 33 seconds. When $1 \mathrm{~m} l$ of water was slowly injected for $\sim 5$ seconds, the initial peak was $134 \%$, and the

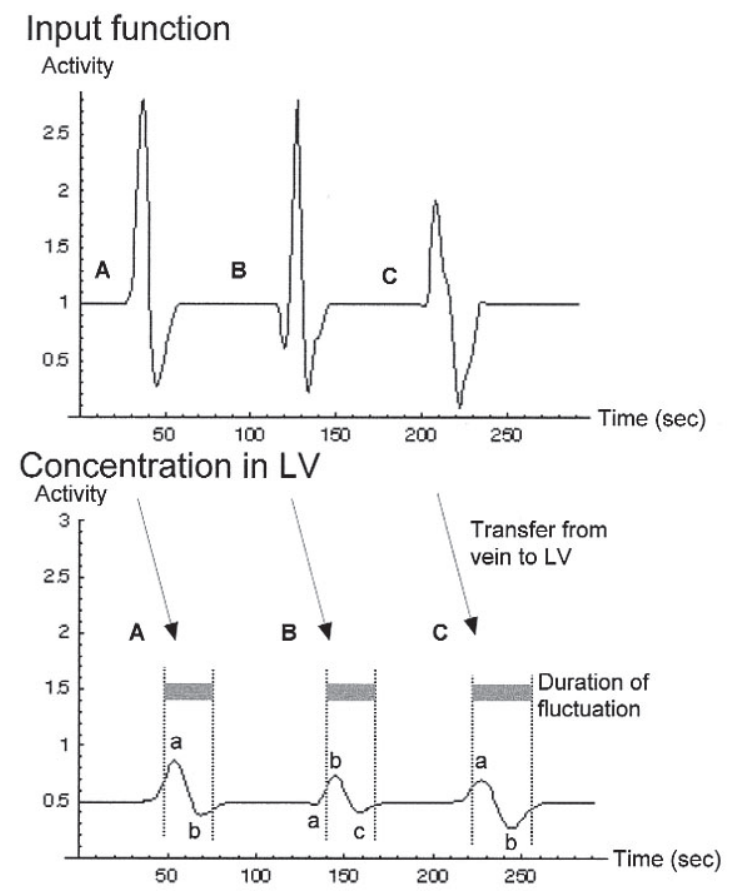

Fig. 3 Typical input function and calculated concentration in LV. A, injection with three ways opened; B, injection with two ways opened; $\mathrm{C}$, slow injection with three ways opened. The peaks $\mathrm{a}, \mathrm{b}$ and $\mathrm{c}$ indicate the first, second and third peaks. The arrows indicate the delay of time-activity curves from the vein to LV. Duration of fluctuation was defined as the interval, when $>20 \%$ or $<-20 \%$ changes are observed (shaded areas). 
second negative peak was low (53\%) with a whole fluctuation time for 31 seconds.

Experiment 3: Volume and modes of injection with two ways opened

With two ways opened, ${ }^{99 \mathrm{~m}} \mathrm{Tc}$ co-injection temporarily interrupted the flow by switching the stopcock. The input function showed a small negative peak, then a positive peak from an increased concentration, followed by a small negative peak (Fig. 3B). The effect of fluctuation in $\mathrm{LV}$ was less with $<2 \mathrm{~m} l$ of water but was $70 \%$ with $3 \mathrm{ml}$ of water injection. The second peak showed a 135 to $160 \%$ increase in LV. The total time of fluctuation was 9 to 25 seconds.

Experiment 4: Flushing with physiological saline

When $10 \mathrm{~m} l$ of physiological saline was used for flushing ${ }^{99 \mathrm{~m}} \mathrm{Tc}$, the input function showed an initial increase followed by a negative peak. The first peak increased significantly with half-lives of 10,5,2 and 1 seconds assumed as shown in Table 2. If the adenosine half-life was assumed to be 2 seconds, flushing of ${ }^{99 \mathrm{~m} T c}$ caused a rapid arrival to LV, which caused a high concentration to $327 \%$ lasting for 25 seconds. If it was assumed to be 1 second, the flushing caused a very high concentration to $1354 \%$ for 34 seconds.

\section{DISCUSSION}

The present study estimated fluctuation of adenosine concentration in the heart when the modes of adenosine infusion and co-injection of myocardial perfusion imaging agents were modified. If the same injection route had been used for constant adenosine infusion and ${ }^{99 \mathrm{~m}} \mathrm{Tc}$ radiopharmaceuticals co-injection, it would have resulted in a 2 to 3 -fold increased concentration in the vein just after the injection site. When a three-way stopcock was used with three ways opened during ${ }^{99 \mathrm{~m}} \mathrm{Tc}$ radiopharmaceutical co-injection, the fluctuation of peak in the LV was relatively low but lasted longer. This sort of fluctuation was intuitively anticipated; however, the actual degree of fluctuation was not evaluated, partly because the adenosine concentration, whose half life is $<10$ seconds, ${ }^{5,6}$ could not be directly measured in LV.

\section{Modes of adenosine injection and fluctuation in concen- tration}

The fundamental kinetic study showed that adenosine concentration reached a plateau 1 minute after injection and returned to a pre-injection condition after 1 minute. Actual coronary dilatation in response to increased adenosine and cessation might have been delayed. In an experimental study with dogs, coronary dilation reached a peak 3 minutes after injection and returned to the preinjection condition 5 minutes later. ${ }^{7}$ Thus, the response of dilatation seemed to be delayed for approximately 3 minutes. The data from the manufacturer showed that duration required for coronary dilatation was $<1 \mathrm{~min}$ in half of the cases and $<2 \mathrm{~min}$ for all cases with injection at $0.120 \mathrm{mg} / \mathrm{min} / \mathrm{kg}$. The disappearance of coronary dilatation is $<1 \mathrm{~min}$ in $1 / 3$ and $<2 \mathrm{~min}$ in $3 / 4$ of the cases. In contrast, some data showed that coronary dilatation was observed even in a 15 second period. Thus, the time required for coronary dilatation may depend on the doses and experimental protocols. When a radiopharmaceutical is injected in a usual manner, adenosine injection was temporarily interrupted with a rotation of the three-way stopcock. The time for interruption depends on the volume of injection, probably from 1 to several seconds, resulting in a decrease in the adenosine concentration in the vein and LV. To avoid the interruption, the radiopharmaceutical can be injected with three-ways opened. In our case, this caused a $+60 \%$ to $+80 \%$ increase in concentration which lasted for 20 to 30 seconds, followed by a decrease for 20 to 30 seconds. A slow injection has been useful to decrease the peak in fluctuation, although the duration of fluctuation was still long. An injection of a small volume was also important to reduce the degree of fluctuation, preferably to less than $1 \mathrm{ml}$. The flushing of radiopharmaceutical with $10 \mathrm{~m} l$ of physiological saline as usually performed in radionuclide angiography shortened the time to arrive at LV as a bolus, and the effect was greater when the half-life of adenosine was assumed to be shorter. Thus the flushing is inappropriate particularly with assumption of a short half-life.

The speed of adenosine injection was recommended to be $0.120 \mathrm{mg} / \mathrm{min} / \mathrm{kg}$. We usually took $0.24 \mathrm{ml} / \mathrm{kg}$ of adenosine and diluted it with physiological saline to obtain $20 \mathrm{~m} l$ of solution. Then the constant infusion rate of $200 \mathrm{~m} / /$ hour $(0.056 \mathrm{~m} / / \mathrm{sec})$ was used. In our clinical situation, the three-way stopcock was placed in the nearest position to the vein between the tube of the butterfly needle and an extension tube. With this condition, the volume of adenosine in a tube of the butterfly needle was approximately $0.5 \mathrm{~m} l$. When $0.5 \mathrm{~m} l$ of adenosine was flushed with a radiopharmaceutical rapidly for 1 second, the volume corresponding to 9 seconds $(0.5 / 0.056)$ was injected rapidly. Even if we had injected $0.5 \mathrm{~m} l$ of the radiopharmaceutical for 9 seconds with three ways opened, the adenosine concentration in the tube would have become $1 / 2$, and the passage would have been twice as fast. Our experiment showed that the actual increase in the injection site was 2 to 3 -fold in concentration and not 9fold. In any case, we can understand that the fluctuation of volume and time can significantly influence the concentration in the vein and heart. Actual variation in coronary flow is influenced by changes in venous return, respiratory movement, condition of vessels in the peripheral vein and lung vasculatures, and other factors. Kajiya et al. studied the stability of coronary flow and the variation was $\pm 20 \%$ in $62 \%$ of cases with infusion rate of $0.12 \mathrm{mg} / \mathrm{m} / /$ $\min .{ }^{8}$ Our study also supported the findings that concen- 
tration was readily influenced by the hemodynamic changes.

\section{Adverse effects of adenosine}

Adverse reactions were observed in approximately $81 \%$ of 9256 consecutive patients, including minor and welltolerated side effects as well as chest pain caused by ischemia. ${ }^{9}$ However, transient atrio-ventricular block occurred in 706 patients and myocardial infarction in 1, severe broncospasm in 7 and pulmonary edema in 1 . Although most of these reactions were generally well tolerated and benign, caution should be observed during adenosine injection to avoid serious effects. As to the route of injection, injection through one infusion line was associated with a slightly higher incidence of side effects, compared with two intravenous lines for adenosine and radiopharmaceuticals. ${ }^{10,11}$ When a single-port infusion protocol was compared with the double-port infusion protocol, the former had a higher incidence of chest pain (57\% vs. $44 \%)$, ST-segment depression (25\% vs. 9\%), nausea (11\% vs. $4 \%)$ and second- or third-degree atrioventricular block ( $11 \%$ vs. $5 \%$ ) in comparison to patients in the latter group. ${ }^{11}$ Since both protocols showed similar detectability for ischemia, they can be used in routine diagnostic protocols.

\section{Limitations}

The present study evaluated fluctuations in LV but not coronary dilatation directly. Therefore, the relationship between coronary dilatation and concentration in LV and the delay in response should be evaluated by new experimental protocols, although continuous measurements of the fluctuation of concentration in LV and actual coronary flow are not easy, particularly in humans. In addition, the relationship between the appearance of adverse effects and adenosine concentration has not been determined and probably depends on the individual patient. This study evaluates input function, transfer of the radionuclide and the possible fluctuations in the ventricles, and the kinetic methodology may be applied to other radiopharmaceutical studies.

\section{CONCLUSION}

Intravenous injection through a single route is feasible, but the changes in concentration are significantly influenced by the modes of injection. The recommended administration through one intravenous line is as follows: The three-way stopcock should be placed in the nearest position to the vein. The volume of injection should be small, and the injection should be slow so as not to displace the adenosine in the infusion line which can reduce the fluctuation. The myocardial imaging agent should be co-injected with three ways open, when a threeway stopcock is used. Adenosine and myocardial imaging agent should not be flushed with physiological saline.
Careful administration during adenosine injection, even with a single injection line, could minimize the fluctuation.

\section{REFERENCES}

1. Klocke FJ, Baird MG, Lorell BH, Bateman TM, Messer JV, Berman DS, et al. ACC/AHA/ASNC guidelines for the clinical use of cardiac radionuclide imaging - executive summary: a report of the American College of Cardiology/ American Heart Association Task Force on Practice Guidelines (ACC/AHA/ASNC Committee to Revise the 1995 Guidelines for the Clinical Use of Cardiac Radionuclide Imaging). J Am Coll Cardiol 2003; 42: 1318-1333.

2. Nishimura S, Nishimura T, Yamazaki J, Doi O, Konishi T, Iwasaki T, et al. Comparison of myocardial perfusion imaging by thallium-201 single-photon emission computed tomography with SUNY4001 (adenosine) and exercise - crossover clinical trial at multi-center. KAKU IGAKU (Jpn J Nucl Med) 2004; 41: 143-154.

3. Sakata Y, Nishimura T, Yamazaki J, Nishimura S, Kajiya T, Kodama K, et al. Diagnosis of coronary artery disease by thallium-201 myocardial scintigraphy with intravenous infusion of SUNY4001 (adenosine) in effort angina pectoris-the clinical trial report at multi-center: phase II. KAKU IGAKU (Jpn J Nucl Med) 2004; 41: 123-132.

4. Yamazaki J, Nishimura T, Nishimura S, Kajiya T, Kodama $\mathrm{K}$, Kato K. The diagnostic value for ischemic heart disease of thallium-201 myocardial scintigraphy by intravenous infusion of SUNY4001 (adenosine) - the report of clinical trial at multi-center: phase III. KAKU IGAKU (Jpn J Nucl Med) 2004; 41: 133-142.

5. Klabunde RE. Dipyridamole inhibition of adenosine metabolism in human blood. Eur J Pharmacol 1983; 93: 2126.

6. Moser GH, Schrader J, Deussen A. Turnover of adenosine in plasma of human and dog blood. Am J Physiol 1989; 256: C799-806.

7. Hidaka T, Yamaki A, Takagi H, N. I, Ohno T. Effect of SUNY 4001 on coronary blood flow in anesthetized openchest dogs [in Japanese, also appeared in the pharmaceutical interview form of Daiichi Pharmaceutical Co., June 2005]. Pharma Medica 2003; 21: 161-167.

8. Kajiya T, Kurogane H, Kijima M, Aizawa T, Tanaka T, Yamazaki J, et al. The effect of continuous infusion on coronary blood flow velocity of patients with effort angina pectoris: comparison between normal and severely stenotic arteries in the same patients [in Japanese]. Kokyu to Junkan 2004; 52: 529-535.

9. Cerqueira MD, Verani MS, Schwaiger M, Heo J, Iskandrian AS. Safety profile of adenosine stress perfusion imaging: results from the Adenoscan Multicenter Trial Registry. $J$ Am Coll Cardiol 1994; 23: 384-389.

10. Korkmaz ME, Mahmarian JJ, Guidry GW, Verani MS. Safety of single-site adenosine thallium-201 scintigraphy. Am J Cardiol 1994; 73: 200-204.

11. Cave V, Heo J, Cassel D, Iskandrian B, Iskandrian AS. Side effects during adenosine thallium imaging with single-port or double-port infusion protocols. Am Heart J 1992; 124: 610-613. 